\title{
ATLAS Tile Calorimeter calibration and monitoring systems
}

\author{
Arely Cortés-González, on behalf of the ATLAS Collaboration \\ European Organization for Nuclear Research (CERN) \\ C/O CERN 1211 Geneve 23, CH \\ Email: arelycg@cern.ch \\ Telephone: (+41) 227673797
}

\begin{abstract}
The ATLAS Tile Calorimeter is the central section of the hadronic calorimeter of the ATLAS experiment and provides important information for reconstruction of hadrons, jets, hadronic decays of tau leptons and missing transverse energy. This sampling calorimeter uses steel plates as absorber and scintillating tiles as active medium. The light produced by the passage of charged particles is transmitted by wavelength shifting fibres to photomultiplier tubes, located in the outer part of the calorimeter. Neutral particles may also produce a signal after interacting with the material and producing charged particles. The readout is segmented into about 5000 cells, each of them being read out by two photomultipliers in parallel. To calibrate and monitor the stability and performance of each part of the readout chain during the data taking, a set of calibration systems is used. This comprises Cesium radioactive sources, Laser, charge injection elements and an integrator based readout system. Information from all systems allows to monitor and equalise the calorimeter response at each stage of the signal production, from scintillation light to digitisation. Calibration runs are monitored from a data quality perspective and used as a cross-check for physics runs. The data quality efficiency achieved during 2016 was $98.9 \%$. These calibration and stability of the calorimeter reported here show that the TileCal performance is within the design requirements and has given essential contribution to reconstructed objects and physics results.
\end{abstract}

\section{INTRODUCTION}

The Tile Calorimeter (TileCal) is the central section of the hadronic calorimeter of the ATLAS experiment [1]. Tile Calorimeter [2] covers the $|\eta|<1.7$ region of the detector. It consists of one barrel and two extended barrel sections and surrounds the Liquid Argon (LAr) barrel electromagnetic and endcap hadronic calorimeters, as seen in Figure 1. The Long Barrel (LB) and Extended Barrel (EB) sections roughly correspond to $|\eta|<1.0$ and $|\eta|>1.0$, respectively. The crack region between the TileCal LB and EB and the LAr electromagnetic barrel and hadronic endcap sections are covered with special cells with a reduced size.

The TileCal provides important information for reconstruction of hadrons, jets, hadronic decays of tau leptons and missing transverse energy. This sampling calorimeter uses iron plates as absorber and scintillating tiles as active medium. The calorimeter readout is segmented into about 5000 cells (longitudinally and transversally), each of them being read by two photomultipliers (PMTs). Tile cells and electronics are organised into 4 partitions, LBA and LBC

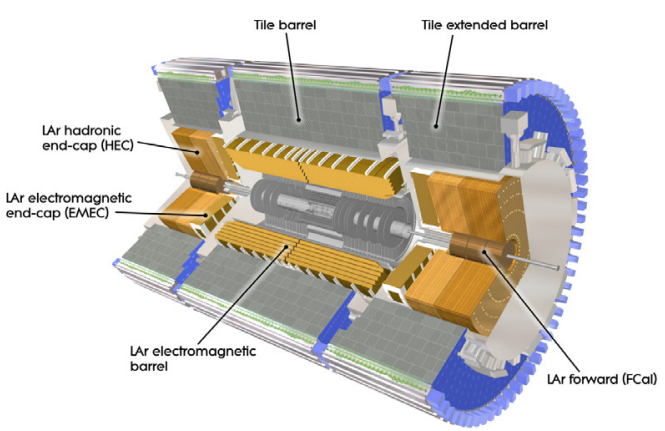

Fig. 1. A cut-away diagram of the ATLAS inner detector and calorimeter. The Tile Calorimeter consists of one barrel and two extended barrel sections [3].

for the $\mathrm{A}$ side and $\mathrm{C}$ side of the barrel region, and separate EBA and EBC partitions in the extended barrel region. A side corresponds to positive $z$ direction, along the beam line. Each partition is divided into 64 symmetric $\phi$ slices (modules), with 45 instrumented channels in LB modules and 32 channels in EB modules. Both LB and EB have up to three layers: the A-layer being the closest to the beam axis, followed by the $\mathrm{B}(\mathrm{C})$ and D-layers. The $\eta$ and radial structure of the TileCal cells is shown in Figure 2a. The cells marked in yellow are the E-cells. They cover the gap/crack region with $1.0<|\eta|<1.6$. They are partly closer to the beam axis than the A-layer cells, thus they are characterised by a larger exposure to the irradiation and by the large amount of light they produce and that is read by the associated PMTs.

The TileCal has three key elements: the scintillators, the PMTs and the read-out electronics. Figure $2 b$ shows a schematic of the assembly of these components. The light is produced in scintillating tiles and converted into electric currents by the PMTs. Their signal is shaped and amplified with two gains. The sampling and the digitisation is realised by ADCs. Trigger signals are formed by an analogue sum of input signals and sent to the calorimeter trigger system, which also considers input from other calorimeters. During collisions, if an event is selected by the trigger system, the digitised signals are collected and processed by a Read-Out Driver. In parallel to this, integrators measure the integrated current from the PMTs. 


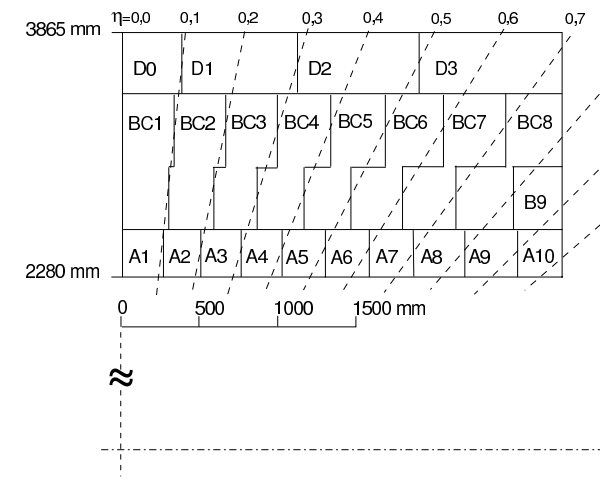

(a)

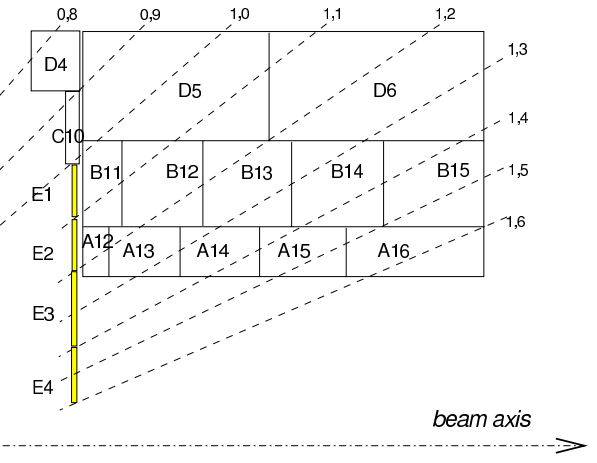

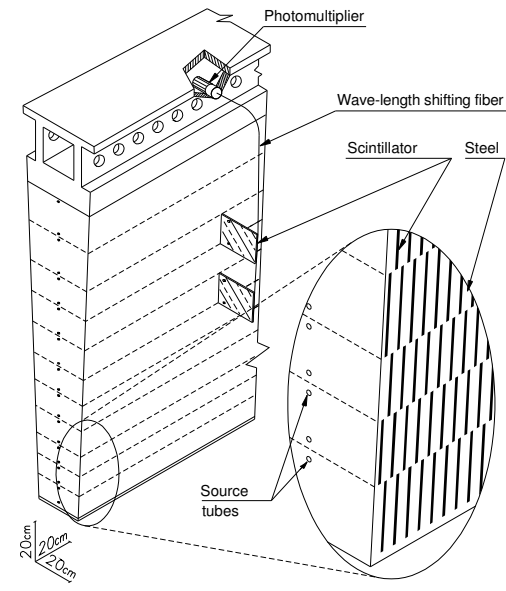

(b)

Fig. 2. (a) TileCal cell and scintillator structure, including the so-called "E-cells" (E1-E4) installed in the gap/crack region, which are highlighted in yellow. (b) Schemetic of the mechanical assembly and the optical readout of the TileCal. The various components of the optical readout, namely the tiles, the fibres and the PMTs, are shown [3].

\section{Calibration Systems}

At each level of the signal development inside the TileCal there is a dedicated calibration system to monitor the behaviour of the different detector components. Figure 3 shows the different calibration systems along with the paths followed by the signals from different sources. Fast readout is used for physics analyses of collision data. The reconstructed energy of each TileCal channel, $E(\mathrm{GeV})$, is derived from the raw response, $A(\mathrm{ADC})$ as follows:

$$
E(\mathrm{GeV})=A(\mathrm{ADC}) \cdot C_{\mathrm{ADC} \rightarrow \mathrm{pC}} \cdot C_{\mathrm{pC} \rightarrow \mathrm{GeV}} \cdot C_{\text {Cesium }} \cdot C_{\mathrm{Laser}}
$$

The factors can evolve in time because of variations of PMTs high-voltage, PMTs stress induced by high light flux or optics ageing. The calibration systems are used to monitor the stability of these elements and provide per channel calibration. While $C_{\mathrm{pC} \rightarrow \mathrm{GeV}}$ was fixed during dedicated test beam campaigns, the remaining calibration constants are provided by individual systems:

- A moveable Cesium radioactive $\gamma$-source to calibrate the optic components and the PMT gains.

- Calibrations of digital gains and linearities with the charge injection system.

- Laser system to monitor the PMTs and the electronic components.

- Monitoring of beam conditions and TileCal optics with the integrator system minimum bias (MB).

\section{A. Cesium Calibration System}

The Cesium calibration system is based on three movable radioactive sources using a hydraulic control. The ${ }^{137} \mathrm{Cs} \gamma$ sources move inside the calorimeter body, emitting $0.662 \mathrm{MeV}$ photons to illuminate the scintillators. The signal generated by the ${ }^{137} \mathrm{Cs}$ source is collected through a special readout that integrates the analog PMT signals. This system is used to calibrate the optical components of the calorimeter as well

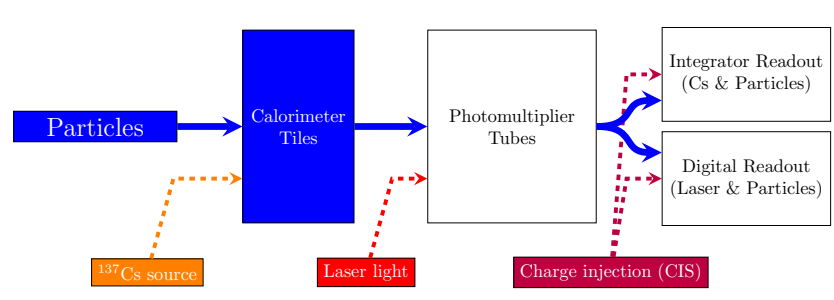

Fig. 3. Flow diagram of the readout signal path of the different TileCal calibration tools. The paths are partially overlapping, allowing for crosschecks and an easier identification of component failures [3].

as the PMTs. The channel response to the energy deposits is used to equalise the response of all the cells and maintain global response of the calorimeter at the electromagnetic scale. Deviation of measured Cesium signals from expected values, corrected for the Cesium decay curve, are interpreted as gain variations and translated into calibration constants $\left(C_{\text {Cesium }}\right)$. From Run 1 to Run 2 of the LHC, a new water storage system, lower pressure in the hydraulics, and more precise water level metering during the scans have been achieved to improve stability and safety of the operation. The deviation of $C_{\text {Cesium }}$ calibration constant from 1 for A-cells (in \%) over the period between 2009 and 2016 is shown in Figure 4. The precision of the system is at the order of $0.3 \%$ with Cesium calibration scans spaced by one to three months up to 2015. During 2016 the frequency was reduced, and scans were taken only at the beginning and end of the proton-proton collisions period. The frequency of the Cesium calibration can be insufficient to track fast drifts of the PMT responses. The Laser system is used between two Cesium scans to correct for this. 


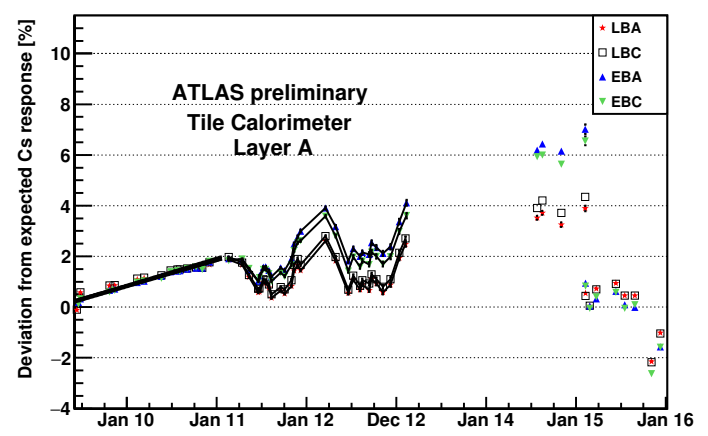

Fig. 4. Variation of Tile Calorimeter response over period of 6 years measured in Cesium calibration runs averaged over all cells in a given partition. The variation is shown for cells in the inner most layer of the calorimeter [3].

\section{B. Charge Injection Calibration System}

A Charge Injection System (CIS) is used to monitor the electronics and extract the conversions factor from ADC counts to $\mathrm{pC}\left(C_{\mathrm{ADC} \rightarrow \mathrm{pC}}\right)$. The CIS simulates physics signals in TileCal channels by injecting a known charge into the ADC and measuring the electronic response. It provides a quantitative relationship between the analog physical signals from the TileCal PMTs and the electronic response of TileCal read-out channels and corrects for non-linearity in the analog signal processing. The corresponding calibration runs are taken from daily to weekly, so $C_{\mathrm{ADC} \rightarrow \mathrm{pC}}$ constants can be regularly produced and applied to data. The overall stability of the calibration factor is at the level of $0.02 \%$ as shown in Figure 5 (for low gain) and usually less than $1 \%$ of the channels exhibit large fluctuations. CIS calibration constants are updated if the deviation from previous measured value is above the CIS systematic error $(0.7 \%)$.

\section{Laser Calibration System}

The gain stability of each PMT is measured using a Laser calibration system [4] that sends a controlled amount of light onto the photocathode of each PMT in the absence of collisions. Deviations of any channel response with respect to its nominal response (at the time of the latest Cesium calibration) is then translated into a calibration constant: $C_{\text {Laser }}$. The Laser calibration runs are usually taken twice a week to monitor the individual PMT gain variations between the Cesium scans. The typical precision on the gain variation during Run 1 was better than $0.5 \%$ per channel. During the LHC Long Shutdown from 2012 to 2015, a new Laser II system was developed to correct shortcomings in electronics and light monitoring of the first system, which in turn resulted in an improved resolution. The new system has been used since the beginning of the Run 2 in 2015 to monitor the channel gain deviations. Figure 6 shows the mean gain variation per cell type between end of May and end of October 2016, covering most of the 2016 proton-proton collisions data taking period. Laser pulses are also sent during empty

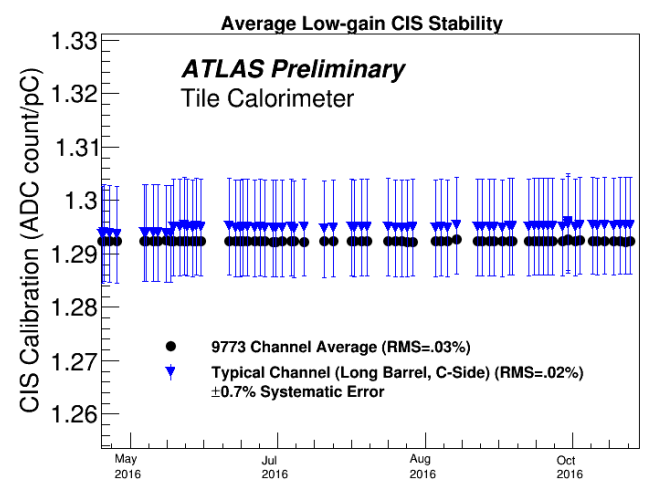

Fig. 5. Detector-wide CIS calibration constant averages of all the low gain channels for each CIS calibration run from 20 April 2016 to 28 November 2016, plotted as black circles. Channels that are unresponsive or have fluctuating CIS constants are not included in the average. The CIS constants from a typical channel (LBC19, channel 13) are additionally plotted as blue triangles for comparison. The RMS values on the plot are indicative of the fluctuation present in calibrations. In addition, there is a $0.7 \%$ systematic uncertainty present in individual calibrations, represented by the blue error bars. High gain channels present similar behaviour [3].

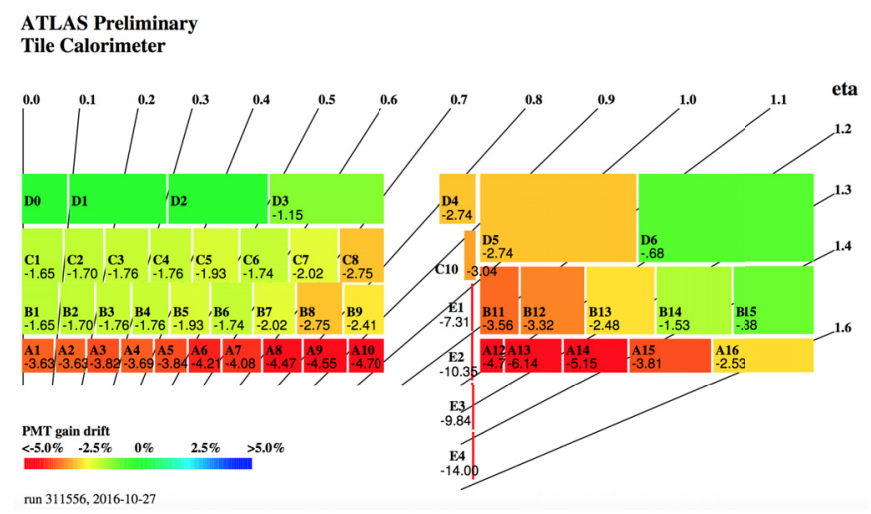

Fig. 6. Mean gain variation (\%) in the TileCal PMTs that read the signal deposited in each channel, as a function of eta and radius, between May 24, 2016 and October 27, 2016. The gain in each PMT is measured using a Laser calibration system. The mean gain variation of the $\sim 10000$ TileCal channels is computed cell by cell. A total of 64 modules in phi are used for each cell while known pathological channels were excluded. The observed down-drift mostly affects cells at inner radius, that are the cells with higher current [3].

bunch crossings of the LHC, with a frequency of $4 \mathrm{~Hz}(1 \mathrm{~Hz})$ during Run 2 (Run 1), and the timing of TileCal response is measured and used for channel per channel time calibration.

\section{Minimum Bias Currents}

High energy proton-proton collisions are dominated by soft parton interactions, also called Minimum Bias (MB) events. The integrator readout is the core of the MB system. It integrates the PMT signal over a large time $(\sim 10 \mathrm{~ms})$, providing a slow control readout. The currents are recorded by all channels of the TileCal. The integrator signal is dominated by $\mathrm{MB}$ events. The fraction of signal coming from physics data events (e.g. containing high $p_{T}$ objects) is extremely negligible. Each collision run is divided into lumiblocks (LBs) 
where a LB is the time interval with a fixed duration where the instantaneous luminosity is about constant. Integrator currents are recorded for each LB unit. The currents are averaged over about 20-25 integrator measurements per LB. Both the Minimum Bias and the Cesium systems measure the signal coming from scintillators and the variation in PMT response over time. Thus, they are expected to give similar results. The MB system can then be used to monitor the stability of the channels and to provide an independent cross-check of the Cesium calibration. The response of the TileCal to signals induced by the MB interactions scale with instantaneous luminosity. Because of this, the system is used to monitor and provide an independent measurement of the ATLAS instantaneous luminosity. The MB response variation measurement is possible by using a reference cell which is known to be stable in time, and which provides a luminosity measurement. The reference cell chosen for the 2016 data studies is cell D6 (outermost layer, covering the region $1.1<|\eta|<1.3$ ) which provides a measurable signal while being relatively protected from irradiation. This reference cell may in fact have a non-zero PMT gain drift, but given that this is well measured with the Laser system, it still serves as a good reference. The comparison between MB and Cesium and the Laser system can give insights on origins of channels drifts (those not related to PMT gain drifts). Figure 7 shows the response variation measured by the MB and the Laser system for cells in the innermost layer of the Extended Barrel, covering the region $(1.2<|\eta|<1.3)$ as a function of time. The integrated luminosity evolution during the same period is also shown in the plot. The variations observed by the MB and Cesium systems are sensitive to PMT gain drift and scintillator irradiation (ageing). The Laser system, however measures only the PMT gain drifts in time. Thus, the difference between MB, or Cesium, and the Laser measurements can be interpreted as a loss of efficiency of the scintillators by irradiation damage. The cell probed in Figure 7, A13, is the most irradiated cell in TileCal, excluding the E-cells.

The comparison of the three calibration systems for the response variation of representative cells has been performed in Run 1 and Run 2 data periods. As mentioned above, the Laser points in Figure 7 show the PMT gain drift, where drops can be observed during collision phase and a rise during machine development phases where no particle collisions take place. Minimum Bias system measures in addition any effect of irradiation of the scintillators when the response variation is higher than the PMT gain drift. While this PMT gain drifts recovers during machine stops, the irradiation effect on the scintillators does not. It is corrected for by adjusting the calibration constants.

Both the integrated luminosity and centre of mass energy $\sqrt{s}$ have an impact on the overall collected charge, $Q$. The charge $Q$ is determined with the help of the luminosity coefficient (exploiting the linear dependance of the current

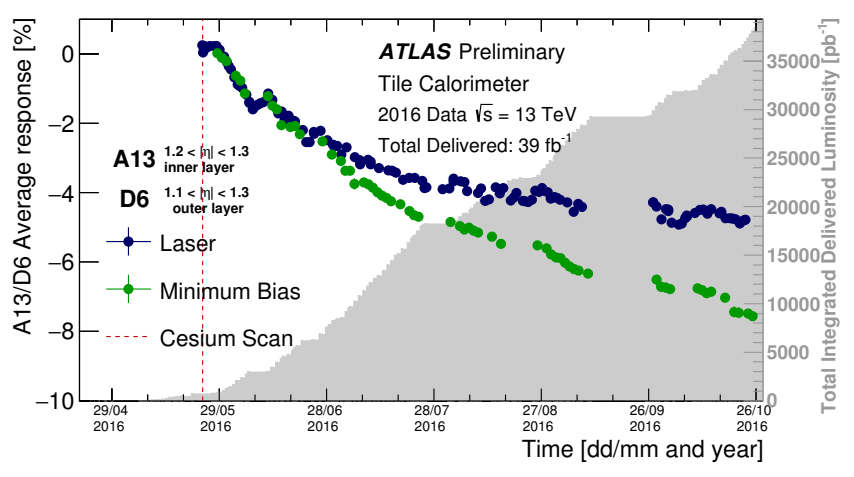

Fig. 7. The variation of the response to Minimum Bias and Laser for cells in the inner layer of the Extended Barrel, covering the region $(1.2<|\eta|<1.3)$, as a function of time. The response variation is derived with respect to a reference cell D6 $(1.1<|\eta|<1.3$, in the outermost layer of the calorimeter). Minimum Bias and Laser data cover the period from the end of May to the end of October 2016. The response variation versus time measured by the Minimum Bias system has been normalised to the response variation measured by the Laser system on May 24th (first point shown in the plot, right after the Cesium scan). The variations observed by the Minimum Bias are sensitive to PMT gain drift and scintillator irradiation. The difference between minimum bias and Laser is interpreted as an effect of the scintillators irradiation [3].

with respect to instantaneous luminosity), $k$ :

$$
Q(t)=k \cdot \int_{t_{0}}^{t} L(t) \mathrm{d} t,
$$

where $t$ is the given time during collisions, and $t_{0}$ is the reference start time. The irradiation effect in scintillators will depend on the collected charge and therefore depends on both the $\sqrt{s}$ and the integrated luminosity. Furthermore, geometry will also play a role, as a larger cell will experience less irradiation effect than a smaller cell with the same collected charge. Figure 8 shows the dependence of the irradiation in cells belonging to the A13 and E-cells in the EB as a function of the collected charge for 2016 data. An irradiation effect of up to $5 \%$ is reported there. The maximum effect is observed for the E3 and E4 cells. A total irradiation effect of at most $3 \%$ for the highest exposed cell has been observed for the 2012 data taking period [5]. For 2015 the largest irradiation effect was below $1 \%$ [6]. The total integrated luminosity in 2015 was $\sim 5$ times lower than the one in 2012. The center of mass energy $\sqrt{s}$ was $8 \mathrm{TeV}$ in 2012 and $13 \mathrm{TeV}$ in 2015 and 2016.

Because of the reduced frequency of the Cesium scans, and the increased integrated charge measured in the system, since the start of 2016, this system is used to correct for the scintillator ageing effect between two Cesium calibrations. This is reflected in the $C_{\text {Cesium }}$ constant in Equation 1.

\section{DATA QuAlity Monitoring}

TileCal monitoring includes identifying and masking problematic channels correcting for timing jumps, monitoring data corruption or other hardware issues. Immediately after 


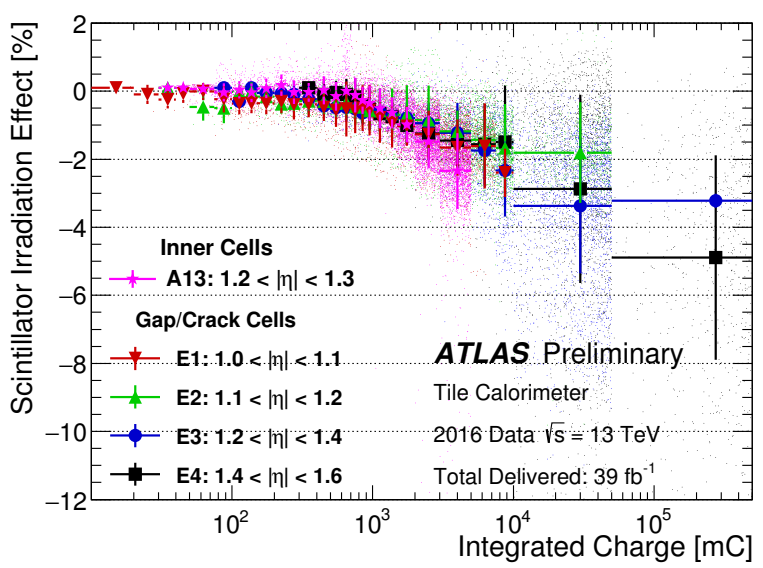

Fig. 8. The relative response variation measured by the Minimum Bias system, after the subtraction of the PMT gain drift component measured by the Laser system, as a function of the integrated charge collected in the cells in the gap/crack region in the Extended Barrel, covering the region $(1.0<|\eta|<1.6)$ and in the cells in the inner layer of the Extended Barrel, covering the region $(1.2<|\eta|<1.3)$, during the period between the end of May to the end of October 2016. The average per cell type is also shown. The integrated luminosity is the total delivered in the whole proton-proton collisions period [3].

a physics run ends, a subset of data (express stream) is processed and reviewed. There is a short delay before the full dataset processing starts, during which any problems identified in the express stream may be corrected with or accounted for in conditions updates. Changes to TileCal timing within collision runs are monitored by Laser calibration events in the empty bunch crossings of physics runs, used to apply timing offset corrections to data. Tile uses a specialised web interface to generate an initial report for a data quality shifter to finish, based on the results of automated data quality monitoring tests. Based on these reviews corresponding actions may be taken. If a problem cannot be corrected, it is entered into the ATLAS Defect database. If the problem is considered intolerable, then the affected data are removed from the ATLAS Good Run List and are not used in physics analyses.

Figure 9 shows the fraction of cells and channels in the detector that have been masked from December 2010. Problematic channels can be recovered by detector interventions during the maintenance periods (hatched areas in the Figure). Figure 10 shows a $(\eta, \phi)$ representation of the number of TileCal masked cells as of June 2017. Tile achieved $99.6 \%$ data quality efficiency in 2012, 100\% in 2015 and $98.9 \%$ in 2016, with $\sim 1 \%$ of cells masked by the end of October, 2016 .

\section{CONCLUSION}

The calibration systems of TileCal are being successfully operated since Run 1 of the LHC. These calibration and monitoring systems have been adapted between Run 1 and Run 2, to adjust to the new LHC conditions. Their

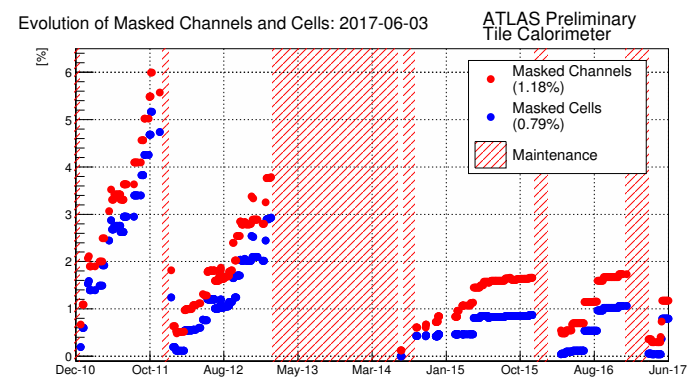

Fig. 9. Percentage of all cells and channels in the detector that are masked as a function of time starting from December 2010. The recent detector status corresponding to the beginning of June 2017 is noted. The hatched area represents the maintenance period of the detector [3].

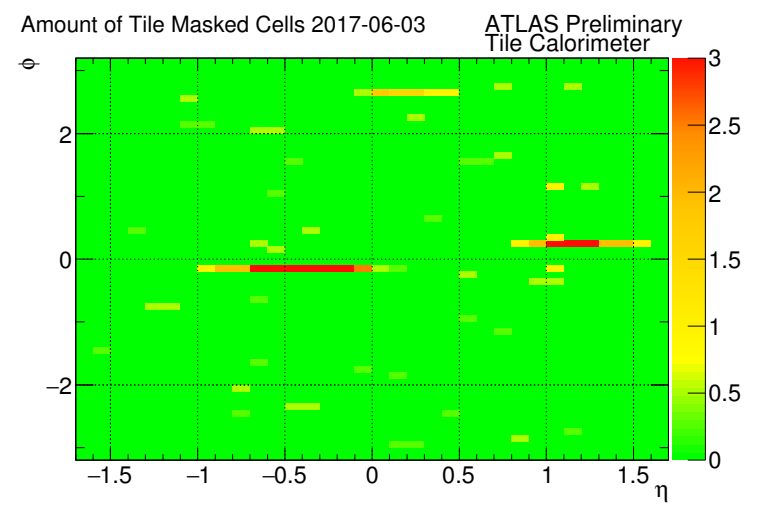

Fig. 10. $(\eta, \phi)$ representation of the number of Tile masked cells. The binning is chosen to be $0.1 \times 0.1$, corresponding to the calorimeter tower definition. Cells in Tile Calorimeter can have one or two readout channels. The bin colour corresponding to integer numbers is the integrated number of cells in the calorimeter tower. Whereas bin colour corresponding to non-integer numbers represents the fraction of masked ADCs in the tower [3].

performances have been reported here. All calibration systems are used to monitor and, when appropriate, correct for instabilities affecting the channels response. These instabilities are understood to be induced by high instantaneous luminosity, optical effects or detector ageing. The monitoring system is able to identify the fraction of pathological channels and have this information propagated to the physics analyses in ATLAS.

\section{REFERENCES}

[1] ATLAS Collaboration, The ATLAS Experiment at the CERN Large Hadron Collider, Journal of Instrumentation 3 S08003, 2008.

[2] ATLAS Collaboration, Readiness of the ATLAS Tile Calorimeter for LHC collisions, Eur. Phys. J. C70: 1193-1236, 2010.

[3] ATLAS Collaboration, https://twiki.cern.ch/twiki/bin/view/AtlasPublic/ApprovedPlotsTile

[4] ATLAS Tile Calorimeter system, The Laser calibration of the Atlas Tile Calorimeter during the LHC run 1, JINST 11 T10005, 2016.

[5] Fracchia, S. Irradiation effect on the response of the scintillators in the ATLAS Tile Calorimeter, Tech. 116 Rep. ATL-TILECAL-PROC-2014013 CERN Geneva, 2014. https://cds.cern.ch/record/1710463

[6] Fischer, C. Study of TileCal Scintillator Irradiation using the Minimum Bias Integrators, ATL-TILECAL-PROC-2016-006 CERN, Geneva, 2016. https://cds.cern.ch/record/2160267 